\title{
Detection of Dental Fluorosis-Associated Quantitative Trait Loci on Mouse Chromosomes 2 and 11
}

\author{
Eric T. Everett ${ }^{a, b}$ Dong Yan ${ }^{c}$ Marjorie Weaver ${ }^{d}$ Lixiang Liu $^{d}$ Tatiana Foroud ${ }^{d}$ \\ E. Angeles Martinez-Mier ${ }^{\mathrm{e}}$

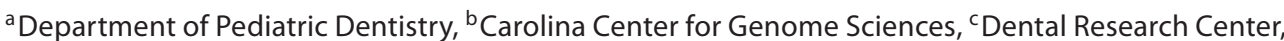 \\ University of North Carolina, Chapel Hill, N.C.; ${ }^{\mathrm{d}}$ Department of Medical and Molecular Genetics and \\ e Oral Health Research Institute, Indiana University Schools of Medicine and Dentistry, Indianapolis, Ind., USA
}

\section{Key Words}

Genetics $\cdot$ Dental fluorosis $\cdot$ Fluoride $\cdot$ Mouse models

\begin{abstract}
Systemic exposure to greater than optimal fluoride (F) can lead to dental fluorosis (DF). Parental A/J (DF-susceptible) and 129P3/J (DF-resistant) inbred mice were used for histological studies and to generate F2 progeny. Mice were treated with 0 or $50 \mathrm{ppm} \mathrm{F}$ in their drinking water for 60 days. A clinical criterion (modified Thylstrup and Fejerskov categorical scale) was used to assess the severity of DF for each individual F2 animal. Parental strains were subjected to histological examination of maturing enamel. $F$ treatment resulted in accumulation of amelogenins in the maturing enamel of $A / J$ mice. Quantitative trait loci (QTL) detection was performed using phenotypic extreme F2 animals genotyped for 354 single nucleotide polymorphism-based markers distributed throughout the mouse genome followed by $\chi^{2}$ analysis. Significant evidence of association was observed on chromosomes 2 and 11 for a series of consecutive markers $(p<0.0001)$. Further analyses were performed to examine whether the phenotypic ef-
\end{abstract}

fects were found in both male and female F2 mice or whether there was evidence for gender-specific effects. Analyses performed using the markers on chromosomes 2 and 11 which were significant in the mixed-gender mice were also significant when analyses were limited to only the male or female mice. The QTL detected on chromosomes 2 and 11 which influence the variation in response to fluorosis have their effect in mice of both genders. Finally, the QTL in both chromosomes appear to have an additive effect.

Copyright $\odot 2008$ S. Karger AG, Basel

\section{KARGER}

(C) 2008 S. Karger AG, Base

Fax +41613061234

E-Mail karger@karger.ch

www.karger.com www.karger.com/cto

\section{Abbreviations used in this paper}

DF dental fluorosis

EDTA ethylenediaminetetraacetic acid

$\mathrm{F} \quad$ fluoride

NBF neutral buffered formalin

QTL quantitative trait loci

SNP single nucleotide polymorphism 
0
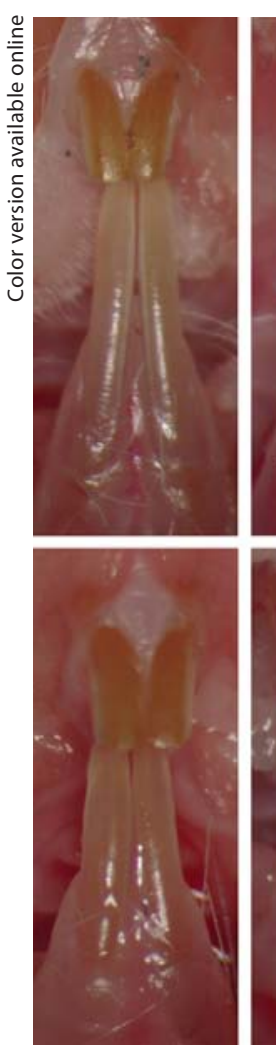

Fig. 1. Clinical images of incisors from DF-susceptible A/J and DF-resistant 129P3/J mouse strains. Panels on the left are from mice treated 60 days with $0 \mathrm{ppm} F$ and panels on the right are from mice treated with $50 \mathrm{ppm}$ F.

\section{Introduction}

Dental (enamel) fluorosis (DF) is an undesirable developmental defect of tooth enamel attributed to greater than optimal systemic fluoride (F) exposure during critical periods of amelogenesis. DF is characterized by increased porosity (subsurface hypomineralization) with a loss of enamel translucency and increased opacity [Fejerskov et al., 1990]. It is generally accepted that increasing DF severity correlates with increasing F exposure. However, individual variation in DF severity can exist when $\mathrm{F}$ exposure is relatively constant in a community [Mabelya et al., 1994; Yoder et al., 1998]. Our studies using inbred strains of mice indicate that genetic background plays a role in DF susceptibility and in F actions on bone biology [Everett et al., 2002; Vieira et al., 2005; Mousny et al., 2006; Yan et al., 2007]. The mechanism(s) that underlie the development of DF remain elusive, as well as the pre- cise stage of amelogenesis most affected by F. Despite the ability of $\mathrm{F}$ to interact at a physicochemical manner with tooth enamel, the cellular target is the ameloblast [Pergolizzi et al., 1995]. In chronic F exposure, the maturation phase of enamel formation appears to be the target stage [Den Besten, 1986; Richards et al., 1986; Den Besten and Thariani, 1992]. Retention of enamel matrix proteins due to reduced removal during enamel maturation, perturbation of extracellular transport or initiation of endoplasmic reticulum stress response pathway indicate that some developmental process is possibly adversely affected by excessive F [Den Besten, 1986; Den Besten and Thariani, 1992; Matsuo et al., 1996; Den Besten et al., 2002; Kubota et al., 2005].

\section{Material and Methods}

\section{Animals}

Male and female A/J (DF-susceptible) and 129P3/J (DF-resistant) inbred mice were obtained from the Jackson Laboratory (Bar Harbor, Me., USA) at 5-6 weeks of age and were acclimated for 1 week prior to mating in reciprocal crosses to produce $\mathrm{F} 1$ hybrids. A panel of F2 mice was produced via sister:brother mating of F1 mice. At weaning, each F2 animal was placed on $\mathrm{NaF}$ provided in the drinking water at a concentration of 50 ppm F ion for 60 days. Parental A/J and 129P3/J mice at 3 weeks of age were treated with 0 or $50 \mathrm{ppm} \mathrm{F}$ as controls for examiner calibration and for histological studies. The treatment duration permitted full development of DF in the erupting incisors (fig. 1) [Everett et al., 2002]. All animals were housed in the Division of Lab Animal Medicine facility within the Dental Research Center, a fully AAALAC-accredited unit, and were maintained on a 12:12-hour light/dark cycle with an ambient temperature of $21^{\circ} \mathrm{C}$. Mice were fed a constant nutrition (LabDiet ${ }^{\circledR}$ 5001; PMI Nutrition International, Richmond, Ind., USA), which contained $0.95 \%$ calcium, $0.67 \%$ phosphorus, $4.5 \mathrm{IU} / g$ vi$\operatorname{tamin} \mathrm{D}_{3}$ and an average $\mathrm{F}$ of $6.56 \pm 0.28 \mu \mathrm{g} / \mathrm{g}$. All experimental procedures were approved by the Institutional Animal Care and Use Committee at the University of North Carolina at Chapel Hill.

\section{Sample Collection}

Serum was collected from each mouse following euthanasia and then frozen at $-80^{\circ} \mathrm{C}$. Samples were randomly selected for $\mathrm{F}$ determinations. Microdirect analysis of serum $\mathrm{F}$ was performed according to the method of Vogel et al. [1990].

\section{DF Phenotyping}

All mice were uniquely identified, and the 2 examiners (E.T.E. and D.Y.) performed independent assessment of DF status for each animal. The determination of DF was made clinically over the entire lower incisor tooth surfaces according to a modified Thylstrup and Fejerskov (TF) index (table 1) [Thylstrup and Fejerskov, 1978; Fejerskov et al., 1994; Everett et al., 2002]. 
Table 1. Classification of the characteristic clinical appearance of fluorotic incisor enamel in mice as modified from Thylstrup and Fejerskov [1978]

TF score 0 The normal translucency of the glossy creamy yellow enamel remains after wiping and drying of the tooth surface.

TF score 1 Thin white opaque lines are seen running across the tooth surface. Such lines are found on all parts of the surface. The lines correspond to the position of the perikymata.

TF score 2 The opaque white lines are more pronounced and frequently merge to form small cloudy areas scattered over the whole surface.

TF score 3 Merging of the white lines occurs, and cloudy areas of opacity spread over many parts of the surface. In between the cloudy areas, white lines can also be seen.

TF score 4 The entire surface exhibits a marked opacity or appears chalky white. Parts of the surface exposed to attrition or wear may appear to be less affected.

From Thylstrup and Fejerskov [1978] and Fejerskov et al. [1994].

\section{Genotyping}

Genomic DNA were prepared using the Puregene Tissue kit (Gentra Systems, Minneapolis, Minn., USA) and quantitated by picogreen fluorometry. Genotyping was performed on the Illumina platform at the Mutation Mapping and Developmental Analysis Project (Brigham and Women's Hospital, Harvard Medical School) [Moran et al., 2006]. Each mouse was genotyped for 354 single nucleotide polymorphism (SNP)-based markers distributed throughout the mouse genome.

\section{Histology}

Prior to euthanasia, mice were perfused with $10 \%$ neutral buffered formalin (NBF) while under anesthesia. Tissues were collected and further fixed in ice-cold NBF overnight followed by demineralization in $0.2 \mathrm{M}$ EDTA pH 7.2 containing $2 \%$ NBF. Mandibles were processed for routine histology. In order to obtain a representative stage of amelogenesis in maturing enamel, cross-sections at the level of the mesial root of the first mandibular molar were obtained and stained with hematoxylin and eosin [Smith and Nanci, 1989]. Serial sections from the same stage of amelogenesis were dewaxed and rehydrated for immunohistochemistry. Following preincubation in 3\% hydrogen peroxide in methanol and rinses in $0.03 \%$ Tween, sections were subjected to primary polyclonal antibodies (rabbit antiporcine amelogenin; gift from Dr. James Simmer, University of Michigan, Ann Arbor, Mich., USA) or normal serum as a control. This primary antibody has been shown to have cross-reactivity to rodent amelogenins [Uchida et al., 1991]. Anti-amelogenin immunoreactivity was visualized using the Vectastain Elite ABC kit (Rabbit IgG; Vector Laboratories, Burlingame,
Calif., USA) according to the manufacturer's instructions. Sections were counterstained using hematoxylin prior to examination.

\section{Statistical Analyses}

$\chi^{2}$ analysis was performed to compare the genotypic distribution in the 2 groups of phenotypically extreme F 2 mice as well as to examine whether the phenotypic effects were found in male and female mice combined or whether there was evidence for gender-specific effects. Statistical significance was reached when $\mathrm{p} \leq$ 0.05 .

\section{Results}

\section{Histology of Normal and Fluorotic Enamel}

Delayed removal or persistence of matrix proteins was present in the maturing enamel of A/J mice. Hematoxylin and eosin serial cross-sections through the mandibles of $\mathrm{A} / \mathrm{J}$ mice at the level of the mesial root of the first molar reveal the presence of eosinophilic staining material in the enamel zone following EDTA demineralization in both control $(0$ ppm F) and treated (50 ppm F) animals (fig. 2a, b). There was greater abundance in the enamel zone following $\mathrm{F}$ treatment. The 129P3/J mice had an enamel zone that was barren of material with only a small accumulation of proteineaceous material following $\mathrm{F}$ treatment (fig. $2 \mathrm{c}$, d). In order to better understand the composition of the accumulated matrix proteins in fluorotic A/J enamel, we performed immmunohistochemistry using anti-amelogenin antibodies. Strong immunoreactivity was found in the enamel zone (fig. 3) and reflected the enamel rod pattern.

\section{Quantitative Trait Loci Identification and Possible Gender Effects}

An F2 panel of 458 mice derived from A/J and 129P3/ $J$ parental strains were treated with 50 ppm $F$ in their drinking water for 60 days. Monitoring of $\mathrm{F}$ exposure, performed by serum $\mathrm{F}$ analyses in randomly selected $\mathrm{F} 2$ animals representing all $4 \mathrm{TF}$ scales, demonstrated a mean serum $\mathrm{F}$ of $12.366 \pm 1.713 \mu \mathrm{M}$. The serum F values obtained in a cross-section of the F2 panel did not significantly differ from serum $\mathrm{F}$ determined in comparably treated parental mice $(11.296 \pm 3.984 \mu \mathrm{M})$. DF severity in the mandibular incisors was assessed in each individual F2 animal using clinical criteria (modified TF scale; table 1). To maximize the power to detect quantitative trait loci (QTL) contributing to the variation in response to DF, only the phenotypic extreme F2 animals (102 

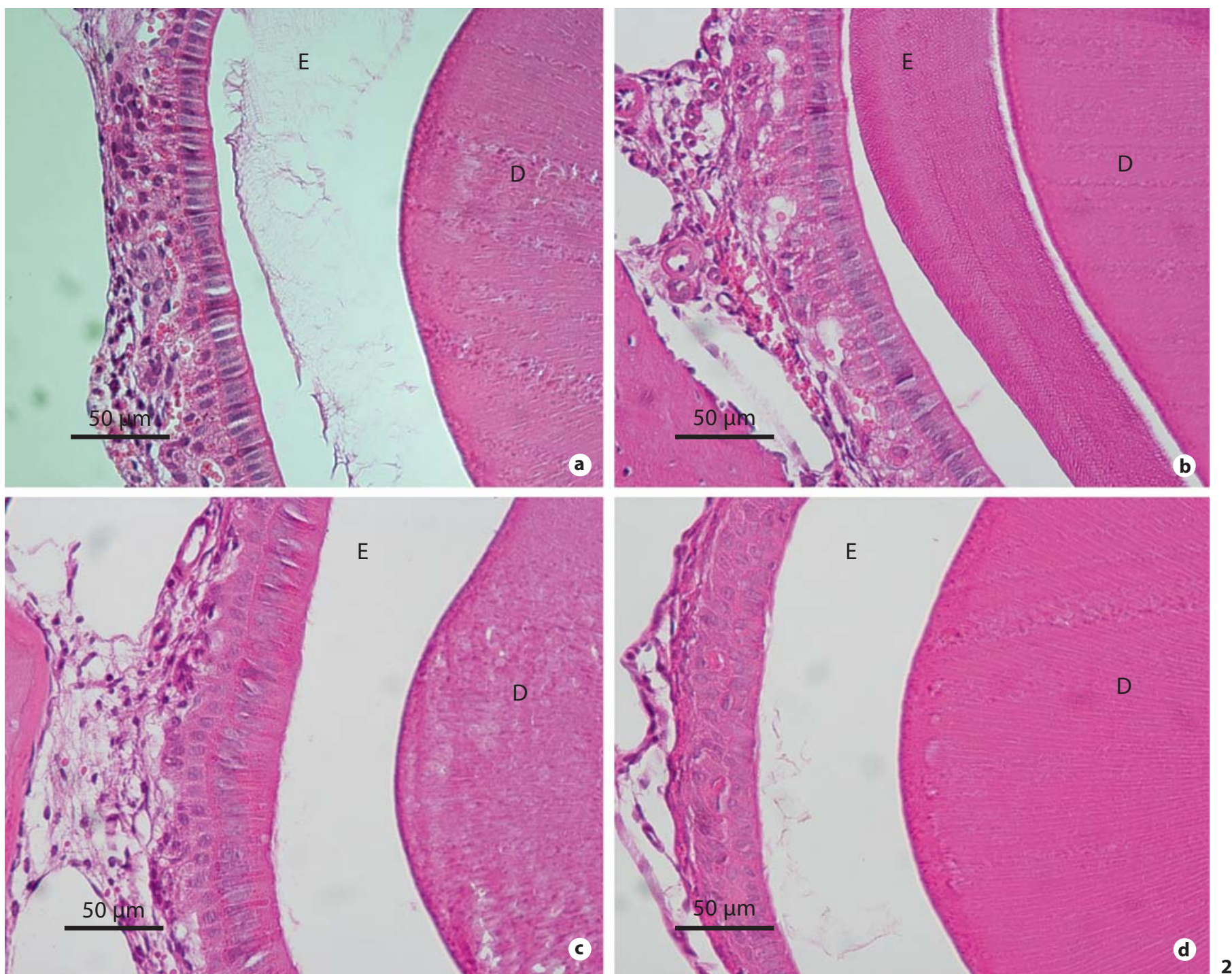

Fig. 2. Hematoxylin and eosin-stained cross-sections of demineralized formalin-fixed paraffin-embedded mandibular incisors at the level of the molar mesial root in A/J (a, b) and 129P3/J (c, d) parental strains treated with $50 \mathrm{ppm} \mathrm{F}(\mathbf{b}, \mathbf{d})$ or $0 \mathrm{ppm} \mathrm{F}(\mathbf{a}, \mathbf{c})$. $\mathrm{E}=$ Enamel zone; $\mathrm{D}=$ dentin.

Fig. 3. Anti-amelogenin immunohistochemical staining of the mandibular incisor enamel zone from an A/J mouse treated with 50 ppm F. Scale bar $=25 \mu \mathrm{m}$.

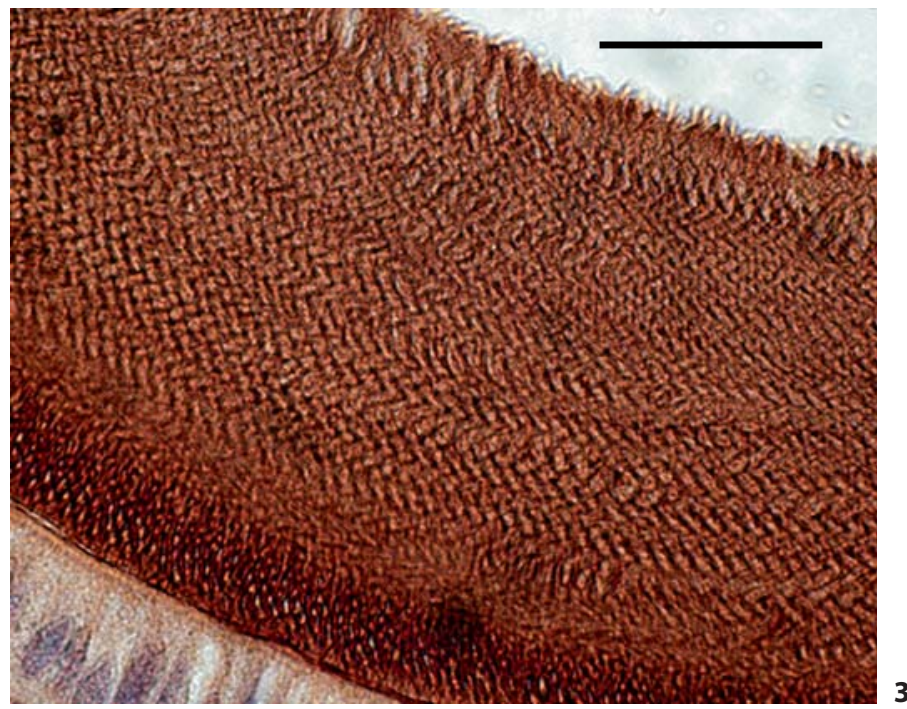


Fig. 4. Genome-wide scan for DF phenotypes for mouse chromosomes 1-19 (excluding $\mathrm{X}$ and $\mathrm{Y}$ chromosomes). The $\mathrm{p}$ values obtained from $\chi^{2}$ analyses were converted to $-\log$ of $\mathrm{p}$ values and plotted on the $y$ axis versus the relative location along each chromosome on the $\mathrm{x}$ axis. Data are from 107 mice representing the phenotypic extremes of animals scored as either a clinical DF of 1 or 4 from a panel of 458 F2 mice.

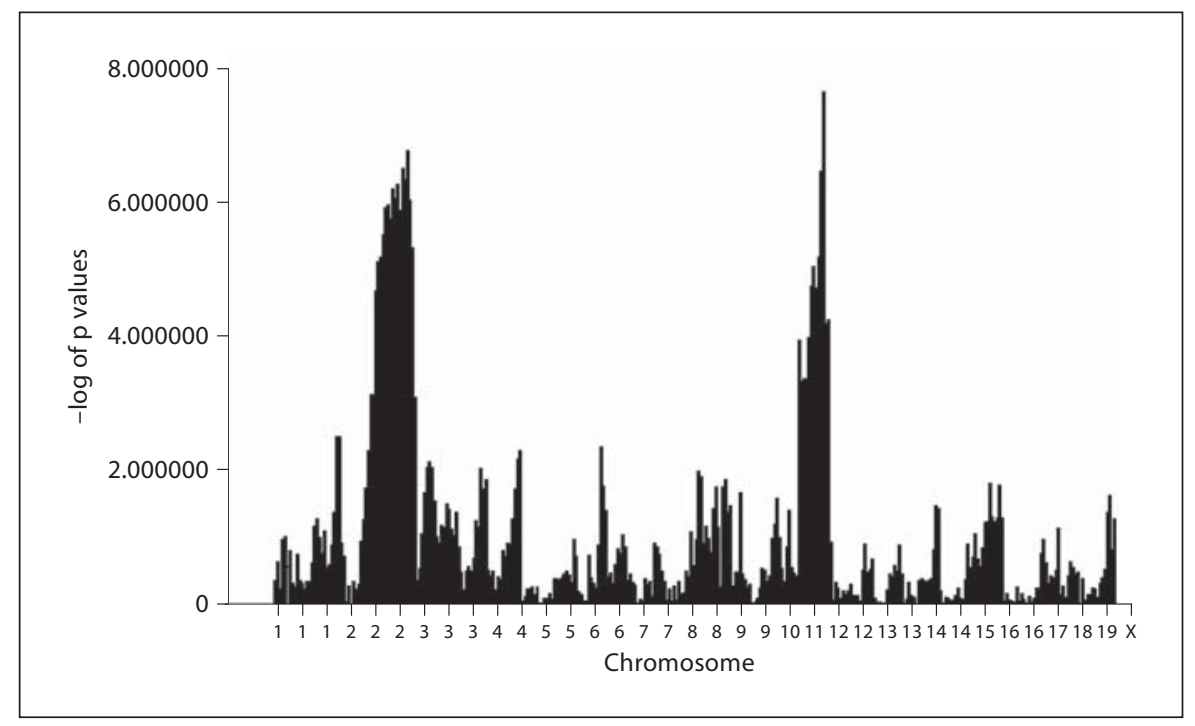

animals with a score of 1 or 4) were genotyped for 354 SNP-based markers distributed throughout the mouse genome. These mice were composed of 55 males and 52 females. $\chi^{2}$ analysis was performed to compare the genotypic distribution in the 2 groups of phenotypically extreme F2 mice. Significant evidence of association was observed on chromosomes 2 and 11 for a series of consecutive markers ( $\mathrm{p}<0.0001$; fig. 4$)$. On chromosome 2 , a QTL interval of approximately $77.62 \mathrm{Mb}$ flanked by markers rs13476589 and UT_2_156.443943 was identified, and on chromosome 11, a QTL interval of approximately $74.29 \mathrm{Mb}$ flanked by markers rs3708339 and rs6161623 was identified.

We observed that female mice represented $74 \%$ of the F2 animals with a TF score of 1 and male mice represented $76 \%$ of the F2 animals with a TF score of 4 . No such gender bias was previously observed with the 2 parental strains or with their F1 hybrid progeny (data not shown). These mice were subjected to analyses to examine whether the phenotypic effects were found in both male and female mice and whether there was evidence for gender-specific effects. Significant gender differences in the frequencies of mild $(\mathrm{TF}=1)$ to severe $(\mathrm{TF}=4)$ $\mathrm{DF}$ in the F2 panel were observed $\left(\chi^{2}, \mathrm{p}<0.0001\right)$. Analyses were performed to examine whether the phenotypic gender-specific effects contributed to possible differences in QTL detection. Analyses performed using the markers on chromosomes 2 and 11 which were significant in the mixed-gender mice were also significant when analyses were limited to only the male or female mice. Therefore, the QTL in these 2 regions which influence the variation in response to DF has its effect in mice of both genders. Finally, preliminary analyses suggest that QTL in both chromosomes appear to have an additive effect.

\section{Discussion}

The objective of the research was to determine what part of the mouse genome carries susceptibility to fluorosis and to investigate further the nature of the enamel defect in the F-susceptible mice. For the former, F-sensitive and F-resistant inbred mice were mated, generating offspring (F2) that were each unique with respect to the mix of chromosomes they inherited from their parents. Their susceptibility to fluorosis was determined by providing them high $\mathrm{F}$ in the drinking water and then staging their fluorosis phenotypes using a modified TF index. As expected, the F2 mice varied with respect to their susceptibility to fluorosis, but unexpectedly, F2 males and females varied in their susceptibility. To characterize what parts of the genome of these mice were inherited from the F-susceptible and F-resistant parents, each F2 mouse was genotyped, that is, characterized for the presence of 354 SNPs distributed throughout the genome. Statistical analyses were performed for the F2 mice that were the most F-resistant and F-susceptible to determine which parts of the genome correlate with these phenotypes. Our investigations of DF susceptibil- 
ity/resistance QTL using A/J and 129P3/J inbred mice in the generation of $\mathrm{F} 2$ progeny was capable of detecting QTL on chromosomes 2 and 11. The QTL intervals detected in this study contain nearly 2,000 genes. More importantly, lack of significant evidence of association observed on chromosomes $\mathrm{X}, 3,5,7$ or 9 suggests a small role of amelogenin, ameloblastin, enamelin, amelotin, Klk-4 or Mmp20 in DF susceptibility/resistance in our animal model. We have no ready explanation for the gender differences observed in our F2 panel. Sex or gender differences in DF affecting humans have not been observed. Future studies will focus on narrowing the QTL intervals detected and identifying candidate genes for further interrogation.

Separately, the nature of the enamel defect in the F-susceptible mice was investigated. Histological analyses of the maturing EDTA-soluble enamel from the DF-susceptible A/J mouse strain following F exposure demonstrated an accumulation of matrix proteins composed in part of amelogenins. Interestingly, some retention of matrix proteins was present in the $0 \mathrm{ppm} F$ treatment group of the A/J strain. The enamel zone of comparably staged 129P3/J incisor remained devoid of any proteinaceous material. The accumulation of enamel matrix proteins in the fluorotic enamel found in the $\mathrm{A} / \mathrm{J}$ mice is consistent with many previous studies [Den Besten, 1986; Den Besten and Thariani, 1992; Den
Besten, 1999]. We found detectable accumulation of matrix proteins in the nonfluorosed enamel of $\mathrm{A} / \mathrm{J}$ mice suggesting that inherent differences in tooth enamel formation may exist between these 2 strains of mice. Previously, we observed baseline differences in enamel quality between A/J and 129P3/J mice. Using microhardness as a criteria, the incisor enamel of $129 \mathrm{P} 3 / \mathrm{J}$ mice is approximately $22 \%$ harder than that of $\mathrm{A} / \mathrm{J}$ [Vieira et al., 2005]. More notably, we found that $F$ treatment (50 ppm F) resulted in a greater loss of enamel microhardness in the $\mathrm{A} / \mathrm{J}$ strain ( $71 \%$ reduction) compared to a $15 \%$ reduction in the $129 \mathrm{P} 3 / \mathrm{J}$ strain. While the retention/persistence of matrix proteins in maturing enamel of $\mathrm{A} / \mathrm{J}$ mice was greatly enhanced by systemic $\mathrm{F}$ exposure and likely contributed directly to the DF phenotype present in this strain, new questions emerge regarding genetically determined variability in normal enamel formation.

\section{Acknowledgements}

The authors are grateful for the technical assistance of Ms. Cynthia Suggs. This work was supported by the National Institutes of Health/National Institute of Craniofacial and Dental Research grant DE014853. Genotyping was subsidized through NIH grants U01 HD43430 and U54 RR020278.

\section{References}

Den Besten, P.K. (1999) Mechanism and timing of fluoride effects on developing enamel. J Public Health Dent 59: 247-251.

Den Besten, P.K. (1986) Effects of fluoride on protein secretion and removal during enamel development in the rat. J Dent Res 65: 1272-1277.

Den Besten, P.K., H. Thariani (1992) Biological mechanisms of fluorosis and level and timing of systemic exposure to fluoride with respect to fluorosis. J Dent Res 71: 1238-1243.

Den Besten, P.K., Y. Yan, J.D. Featherstone, J.F. Hilton, C.E. Smith, W. Li (2002) Effects of fluoride on rat dental enamel matrix proteinases. Arch Oral Biol 47: 763-770.

Everett, E.T., M.A. McHenry, N. Reynolds, H. Eggertsson, J. Sullivan, C. Kantmann, E.A. Martinez-Mier, J.M. Warrick, G.K. Stookey (2002) Dental fluorosis: variability among different inbred mouse strains. J Dent Res 81: 794-798.
Fejerskov, O., M.J. Larsen, A. Richards, V. Baelum (1994) Dental tissue effects of fluoride. Adv Dent Res 8: 15-31.

Fejerskov, O., F. Manji, V. Baelum (1990) The nature and mechanisms of dental fluorosis in man. J Dent Res 69 (Spec No): 692-700; discussion 721 .

Kubota, K., D.H. Lee, M. Tsuchiya, C.S. Young, E.T. Everett, E.A. Martinez-Mier, M.L. Snead, L. Nguyen, F. Urano, J.D. Bartlett (2005) Fluoride induces endoplasmic reticulum stress in ameloblasts responsible for dental enamel formation. J Biol Chem 280: 23194-23202.

Mabelya, L., M.A. van 't Hof, K.G. Konig, W.H. van Palenstein Helderman (1994) Comparison of two indices of dental fluorosis in low, moderate and high fluorosis Tanzanian populations. Community Dent Oral Epidemiol 22: $415-420$.
Matsuo, S., T. Inai, K. Kurisu, K. Kiyomiya, M. Kurebe (1996) Influence of fluoride on secretory pathway of the secretory ameloblast in rat incisor tooth germs exposed to sodium fluoride. Arch Toxicol 70: 420-429.

Moran, J.L., A.D. Bolton, P.V. Tran, A. Brown, N.D. Dwyer, D.K. Manning, B.C. Bjork, C. Li, K. Montgomery, S.M. Siepka, M.H. Vitaterna, J.S. Takahashi, T. Wiltshire, D.J. Kwiatkowski, R. Kucherlapati, D.R. Beier (2006) Utilization of a whole genome SNP panel for efficient genetic mapping in the mouse. Genome Res 16: 436-440.

Mousny, M., X. Banse, L. Wise, E.T. Everett, R. Hancock, R. Vieth, J.P. Devogelaer, M.D. Grynpas (2006) The genetic influence on bone susceptibility to fluoride. Bone 39: 1283-1289.

- Pergolizzi, S., A. Santoro, G. Santoro, F. Trimarchi, G. Anastasi (1995) Enamel fluorosis in rat's incisor: S.E.M. and T.E.M. investigation. Bull Group Int Rech Sci Stomatol Odontol 38: 95-104. 
Richards, A., J. Kragstrup, K. Josephsen, O. Fejerskov (1986) Dental fluorosis developed in post-secretory enamel. J Dent Res 65: 14061409.

Smith, C.E., A. Nanci (1989) A method for sampling the stages of amelogenesis on mandibular rat incisors using the molars as a reference for dissection. Anat Rec 225: 257-266.

-Thylstrup, A., O. Fejerskov (1978) Clinical appearance of dental fluorosis in permanent teeth in relation to histologic changes. Community Dent Oral Epidemiol 6: 315-328.
Uchida, T., T. Tanabe, M. Fukae, M. Shimizu, M. Yamada, K. Miake, S. Kobayashi (1991) Immunochemical and immunohistochemical studies, using antisera against porcine 25 $\mathrm{kDa}$ amelogenin, $89 \mathrm{kDa}$ enamelin and the 13-17 kDa nonamelogenins, on immature enamel of the pig and rat. Histochemistry 96 : 129-138.

Vieira, A.P., R. Hancock, H. Eggertsson, E.T. Everett, M.D. Grynpas (2005) Tooth quality in dental fluorosis genetic and environmental factors. Calcif Tissue Int 76: 17-25.

Vogel, G.L., C.M. Carey, L.C. Chow, J. Ekstrand (1990) Fluoride analysis in nanoliter- and microliter-size fluid samples. J Dent Res 69 (Spec No): 522-528; discussion 556-557.
Yan, D., A. Gurumurthy, M. Wright, T.W. Pfeiler, E.G. Loboa, E.T. Everett (2007) Genetic background influences fluoride's effects on osteoclastogenesis. Bone 41: 1036-1044.

Yoder, K.M., L. Mabelya, V.A. Robison, A.J. Dunipace, E.J. Brizendine, G.K. Stookey (1998) Severe dental fluorosis in a Tanzanian population consuming water with negligible fluoride concentration. Community Dent Oral Epidemiol 26: 382-393. 